\title{
Design considerations for an eHealth decision support tool in inflammatory bowel disease self-management
}

Belinda D Jackson, MBBS, BBioMedSci, FRACP ${ }^{1,2}$

Consultant gastroenterologist

Danny Con, BSc, MD

Research fellow

Peter De Cruz, MBBS, PhD, FRACP ${ }^{1,2}$

Consultant gastroenterologist and Senior Lecturer

\section{Affiliations:}

${ }^{1}$ Department of Gastroenterology, The Austin Hospital, Melbourne, Australia

${ }^{2}$ University of Melbourne, Department of Medicine, Austin Academic Centre, Melbourne, Australia

\section{Author for correspondence:}

Dr Peter De Cruz

Department of Medicine, Austin Academic Centre

The University of Melbourne

Victoria Australia 3084

Tel: +61 394965353

Fax: +61394963487

Email:ppdecruz@gmail.com

This is the author manuscript accepted for publication and has undergone full peer review but has not been through the copyediting, typesetting, pagination and proofreading process, which may lead to differences between this version and the Version of Record. Please cite this article as doi: $10.1111 / \mathrm{imj} .13677$

This article is protected by copyright. All rights reserved. 


\section{Acknowledgments}

The Gutsy Group provided research support. Ferring has provided an unrestricted educational grant. AbbVie has provided research support. PDC is supported by a David Bickart Clinician Research award from the University of Melbourne and Bushell Postdoctoral award from the Gastroenterological Society of Australia (GESA). BDJ is supported by a National Health \& Medical Research Council (NHMRC) postgraduate scholarship and AVANT Doctor in Training (DiT) scholarship.

\section{Disclosure of interest and Sources of Funding}

PDC has received educational support, consulted on advisory boards, and been a speaker at educational symposia sponsored by Shire, Ferring, Janssen, Takeda, AbbVie, and Baxter. BDJ has received educational support from Merck Sharp \& Dohme, Aspen, and Ferring. This study was not directly funded.

\section{Word count}

Abstract 248

Main text 2991

This article is protected by copyright. All rights reserved. 


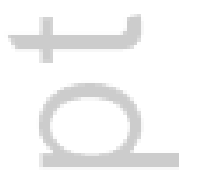




\section{ABSTRACT}

\section{Background}

Electronic health (eHealth) decision support tools have the potential to: facilitate inflammatory bowel disease (IBD) self-management; reduce health care utilization, and; alleviate the pressure on over-burdened outpatient clinics. The purpose of this study was to explore the perspectives of key stakeholders on the potential use of a decision support tool for IBD patients.

\section{Methods}

A qualitative study using focus group methodology was conducted at a tertiary IBD center in Melbourne, Australia in February 2015. Key stakeholders including physicians, nurses, and patients were included in the study. Two independent reviewers undertook inductive coding and generated themes.

\section{Results}

31 participants were included in the study (16 males; 11 physicians, 6 nurses). An eHealth decision support tool was thought to be beneficial to facilitate IBD self-management. Four themes emerged: (1) Framework for the decision support tool - the tool should be an adjunct to current models of care and facilitate shared decision making and patient engagement; (2) Target population - stable patients with mild-to-moderate disease; (3) Functionalities of the intervention - a web-based platform encompassing patient reported outcomes, objective markers of disease and clinical algorithms based on international guidelines, and; ${ }^{1}$ Design and Implementation - patients should be involved in the design.

\section{Conclusions}

EHealth interventions are thought to be an important strategy towards facilitating self-management for patients with IBD. A multi-stage iterative approach should be adopted in the design and implementation process of eHealth interventions. Patient perspectives need to be sought prior to and throughout development of an eHealth decision support tools for IBD. 
Key words: eHealth, focus group, inflammatory bowel disease, selfmanagement, telemedicine 


\section{INTRODUCTION}

Inflammatory bowel diseases (IBD) including Crohn's disease (CD) and ulcerative Colitis (UC) are chronic inflammatory disorders of the gastrointestinal tract that are associated with significant physical and psychological morbidity ${ }^{2-5}$. IBD is characterized by intermittent flares of disease activity requiring adjustments in medication, frequent outpatient clinic visits, hospitalizations and surgeries ${ }^{6}$. The increasing incidence of IBD over the past decade has resulted in increased health care resource utilization and longer specialist outpatient waiting lists ${ }^{4,7,8}$. Moreover, non-metropolitan patients have limited access to specialist IBD outpatient clinics. The burden of IBD on the health care system is likely to increase as the incidence of IBD is rising worldwide and increasingly complex integrated models of health care are required $^{9-12}$.

Electronic health or 'eHealth' technologies represent a potential solution to facilitate distance management of patients and enable earlier intervention compared to traditional outpatient based models of care ${ }^{13}$. The principle underlying the distance/remote management of patients via eHealth is the incorporation of a component of patient self-management ${ }^{14}$ whereby patients relay information about their state of health to a program or health care team, which gives them feedback to support decision making ${ }^{13,15}$. As a significant component of chronic disease management, patient self-management has been shown to improve symptoms, psychological well-being, and health care utilization $^{16}$.

Clinical decision support systems characterize an important field within health informatics and are defined as any computer-based system that aids in clinical decision making ${ }^{17}$. While traditionally their application has been limited to the clinical decision making of medical professionals and hospitals, the incorporation of decision support as eHealth tools to aid self-management in patients with chronic diseases is increasing ${ }^{13}$. Within the IBD community there is growing interest in the development and implementation of decision support tools, where electronic devices such as mobile phones and computers are 
used to analyze patient symptom data with predetermined algorithms to generate real-time management plans ${ }^{18-22}$. EHealth has the potential to augment current models of care and reduce health care utilization by alleviating pressure on overburdened outpatient clinics and hospital emergency departments. Although the field of eHealth is rapidly growing in the IBD setting, new eHealth tools should be developed with a level of scientific rigor comparable to conventional medicine, which requires multiple phases including planning, implementation, evaluation and analysis ${ }^{23,24}$.

The aim of this study was to explore key stakeholders' perceptions regarding a potential eHealth decision support tool for patients with IBD in order to inform and refine the development of a decision support tool for IBD selfmanagement. 


\section{METHODS}

\section{Study design}

The design of this study followed recommended methods for an iterative design process for the development and implementation of eHealth interventions in chronic disease management ${ }^{24,25}$. This study addresses the first step in a multi-stage process, using focus group methodology to explore perceptions of key stakeholders regarding a potential eHealth decision support tool to facilitate IBD self-management. The focus group was conducted in February 2015 at a large tertiary referral center in Melbourne, Australia.

\section{Study participants}

We used a purposeful sampling approach to identify and select study participants who were most relevant to the topic of interest ${ }^{26}$. Sampling was guided by the list of suggested key stakeholders in the design and implementation of eHealth for chronic disease management ${ }^{24}$, which included health professionals (IBD physicians, IBD nurse consultants, general gastroenterologists, a GP liaison nurse and surgeons); a health care organization (a representative from Crohn's Colitis Australia (CCA), the national peak patient body representing Australian patients with UC or CD); medical researchers; an information technology (IT) expert (department head of IT services); and policy makers. Invitations for the focus group were disseminated via email. Written and verbal informed consent was taken prior to commencement of the interviews. No incentives/honoraria for participation in the study were offered.

\section{Proposed decision support tool}

Key stakeholders within the focus group were informed during the focus group meeting that the proposed eHealth intervention was a decision support tool that was intended to facilitate self-management for patients with IBD. The focus group was informed that the intervention would take the form of a computer program that had the potential to be accessed by personal computer, tablet or mobile phone. In particular, the primary functionality of the 
intervention proposed was to be decision support, whereby users would be able to input symptom data that would be processed by a pre-determined algorithm in order to generate automated management advice. The algorithm would be based on the published European Crohn's and Colitis Organisation (ECCO) guidelines to stratify disease activity and the Hospital Anxiety and Depression Scale (HADS) to stratify psychological distress, with an additional functionality that would encompass the preventive aspects of care. The tool would provide alerts using a traffic light system, where a green signal would indicate no disease activity, amber would indicate mild or moderate activity, and red would indicate severe activity. Management advice would include "no action" for a green alert, drug modifications for an amber alert, and immediate contact with the IBD team and possible presentation to the emergency department for a red alert.

\section{Procedures}

The focus group was preceded by structured preliminary briefings on key subject matters and was facilitated by a panel of presenters including the Executive Director of Acute Operations at the hospital, the departmental Director of IBD services (PDC), an IBD research fellow (BDJ) and a specialist IBD psychologist. Preliminary presentations covered the topics of: current hospital-wide eHealth initiatives; current and future models of care in IBD; current eHealth interventions in IBD for disease and psychological selfmanagement, and; the proposed eHealth decision support tool for IBD selfmanagement.

The focus group was moderated by PDC. A semi-structured group interview process was used to collect data whereby a topic guide was pre-specified by expert consensus between the panel members and used by the moderator to direct discussion. Four topics for discussion were included: 1) Framework for the project; 2) Outcome measurements; 3) Patient self-management; and 4) Traction and Sustainability. The moderator began each topic by asking a discussion starter question. The discussion of each topic was ended when no new information was generated through the interview process (the point of saturation). The proceedings were audiotaped, videotaped and scribed. 


\section{Data analysis}

Recordings of the focus group were transcribed for analysis. Two independent reviewers (BDJ and DC) used a thematic analysis approach with an inductive coding scheme to analyse the data ${ }^{27}$. Inter-rater reliability between the two reviewers was quantified by percentage observed agreement and Cohen's kappa coefficient ${ }^{28}$. 


\section{RESULTS}

\section{Participant demographics}

Thirty-one participants were included in the study (see Table 1). Attendees included doctors, nurse consultants, IT specialists and hospital executives amongst others. There was an even spread of males and females.

\section{Thematic analysis}

Four key themes were identified in the qualitative analysis. Key suggestions with exemplar quotes are given in Table 2. Sixty-seven items were identified with 20 potentially applicable codes. Agreement between the two coders was high (95.1\% observed agreement) and inter-rater reliability was in the substantial range (Cohen's $\mathrm{k}=0.64$, SD 1.6, 95\% Cl 0.55-0.72).

\section{Framework for the Decision Support Tool}

Participants discussed their support for and the feasibility of the design and implementation of the eHealth intervention within the suggested framework to facilitate IBD self-management. All participants supported the notion of eHealth strategies as a potential alternative model of care, pending careful consultation, design and evaluation. Participants also discussed participatory medicine as a model of care, where patients take a more active role in their management, leading to shared decision-making and deep patient engagement ${ }^{29}$. It was agreed that eHealth could facilitate participatory medicine as a solution to IBD self-management.

Several concerns were raised about the proposed eHealth intervention for IBD patients. One participant raised the possibility of patients losing their relationship with the IBD clinical team by relying too much on electronic care. However, participants agreed that strict inclusion criteria should apply to the intervention such that only stable patients who have already built a relationship with the clinical IBD team would use the intervention. Participants agreed they would support the proposed decision support tool if it were ensured that every patient would be provided with "meaningful clinical care," 
equivalent or improved quality of care compared to patients' existing care. Despite some participants voicing a degree of concern for the adoption of eHealth to aid disease management, the overarching consensus was that the introduction of eHealth in IBD was timely and necessary.

\section{Target population}

Participants discussed the ideal patient population that should be targeted by the decision support tool. A majority of participants agreed that the eHealth model would be most appropriate to augment or replace outpatient face-toface management of stable and relatively less complex patients as a priority. One participant stated that "strict inclusion and exclusion criteria (should) be used". Stratifying interventions by disease subtype was deemed to be important, and in particular the proposed decision support tool should target patients with mild-to-moderate UC given the simpler management requirements. Chronicity of disease was mentioned briefly, and one participant suggested that the decision support tool be used "only by patients with stable disease and a diagnosis of IBD for at least two years". It was agreed that patients with complex disease such as "obstructive symptoms, recent surgery and those with perianal disease (if CD were included) ought to be excluded" from using the decision support tool. It was felt that the ideal patient for a model of care incorporating eHealth decision support should have mild-to-moderate UC and have minimal disease activity at study entry.

\section{Functionalities of the intervention}

Participants discussed the specific functionalities that the eHealth decision support tool ought to possess. A web-based platform was thought to be more appropriate compared to a mobile phone application for initial testing of the decision support tool. Participants agreed that disease monitoring should form a component of the decision support tool. The recording of patient reported outcomes (PROs), clinical disease indices and laboratory biomarkers in the decision support tool were all considered feasible, but it was agreed that only validated outcome measures should be used. 
A majority of the participants endorsed the use of automated algorithms to facilitate self-management as a primary aim of the decision support tool. It was unanimous amongst the participants that treatment algorithms should be developed from the ECCO guidelines. Several participants raised concerns regarding the appropriateness of the clinical scenarios that should be targeted by the decision support tool. The major concern regarded titration of corticosteroids, where participants agreed that face-to-face contact was more appropriate compared to eHealth decision support. However, selfmanagement of aminosalicylates with decision support, both orally and rectally, was unanimously thought to be appropriate. Furthermore, management of psychological distress was also deemed possible with the proposed decision support tool.

Participants suggested several additional functionalities should be considered in the model of care. Delivery of IBD education was thought to be important and necessary to maximise efficacy of the tool. Access to information regarding chronic disease prevention was thought to provide important additional education to both patients and clinicians. It was agreed that although feasible, social media was not a priority and should not be integrated within the decision support tool.

\section{Design and Implementation}

Participants discussed the implementation, testing and sustainability of the eHealth intervention. It was unanimous that "eHealth in IBD is required," with one participant expressing that "people want this thing already." One participant stated that the intervention needed to be "patient-centered." "Including patients in the design phase" and "conducting focus groups for patients" were suggested to improve implementation of the eHealth intervention. Involvement of general practitioners was also suggested to ensure maximal uptake. Privacy concerns were identified as a potential barrier to implementation. However, the Manager of Strategy and Engagement of IT Services commented that "delivering care outweighs the risk of security" in this setting. Participants agreed with this statement. Methods to minimise the risk of security were discussed and included the 
"minimisation of email content" and "entering more content into the eHealth intervention as soon as it is available". Outcome measures that were raised as being important to include were patient reported outcomes including quality of life scores and frequency of hospital presentations.

Participants raised concerns about the sustainability of the intervention, alluding to the issue of non-adherence to pre-existing eHealth solutions ${ }^{30}$. Methods suggested to improve traction and sustainability included "a visually engaging and interactive tool" that "needed to be sticky" and "slick." A panel member alluded to the fact the "we want the patients to talk about the model." Finally, participants discussed the technical and funding requirements of the eHealth intervention to ascertain the feasibility of the project. Participants agreed that developmental costs of the eHealth intervention may be significant, but cost savings of the intervention might be more substantial. EHealth was therefore considered unanimously to be a worthwhile investment of public health care dollars. 


\section{DISCUSSION}

This study has helped inform the design principles that ought to be considered in an eHealth decision support tool for IBD self-management. Key stakeholders collectively believed that an eHealth decision support tool should aim to augment current IBD outpatient management. The primary utility of the decision support tool should be to provide IBD patients with the tools necessary to safely and effectively manage symptoms and flares of their disease activity, psychological distress and the preventative health aspects of care. Decision support tools ought to be prioritised among stable patients with mild-to-moderate UC. Outcome measures should include both PROs and objective measures of disease activity.

Previous eHealth technologies have aimed to provide general support to IBD patients with interventions such as reminder tools, tools to educate or provide disease information, and tools to record symptoms ${ }^{4,31-34}$. However, decision support tools in IBD are now feasible as current eHealth technologies can provide the necessary components including: functionalities that allow recording of patient reported outcomes and clinical biomarkers; validated disease severity indices on which algorithms can be based, and; a means of automatically delivering treatment suggestions in real-time ${ }^{18-22}$. Validated disease activity indices such as the Crohn's disease activity index (CDAI) and the Simple Clinical Colitis Activity Index (SSCAI) can be reliably completed by patients using eHealth tools to measure disease activity ${ }^{35-37}$. Moreover, homebased measurement of fecal calprotectin is feasible and has been shown to agree with hospital-based enzyme-linked immunosorbent assays (ELISA) ${ }^{38,39}$.

Previous eHealth decision support tools have lacked a multi-stage design process with appropriate consultation, which is necessary given the complex interactions between key stakeholders ${ }^{24,40}$. This may be due to a lack of research in the design and implementation process of eHealth tools ${ }^{24}$. Previous eHealth solutions have lacked professional medical involvement, encountered issues with implementation and non-adherence, and struggled to 
successfully engage users ${ }^{34,41,42}$. A likely explanation is that eHealth modalities which lack a theoretical framework have been produced without proper consultation or involvement of key stakeholders. Care must be taken to address factors such as perceived risk of use, perceived usefulness and degree of user-centric focus, which influence uptake and usage of eHealth technologies ${ }^{43-46}$.

A phased approach should be developed for all interventions, starting with systematic reviews to identify the known literature, followed by theoretical work to establish an appropriate theoretical foundation ${ }^{24,47}$. Modeling studies should follow to identify potential barriers to implementation and population impact, followed by qualitative studies to determine acceptability and feasibility, and concluding with pilot studies to optimise both the intervention and the trial parameters. Only when both the intervention and the trial parameters have been met, should researchers proceed to a controlled trial to establish the effectiveness of the intervention. Evaluating performance at each of these phases is critical to the overall success of the intervention ${ }^{23}$ and to ensure that these systems are safe, beneficial and an appropriate use of limited resources.

While this study facilitated interdisciplinary collaboration to maximise the potential efficacy and uptake of the eHealth intervention, further studies are needed to elucidate the perspectives of patients in IBD. The priorities of doctors and patients may differ, such that physicians tend to favor tools that promote patient compliance in following orders, and patients tend to favor tools that facilitate collaboration and shared decision making ${ }^{48}$. Without proper consultation with both patients and doctors, design flaws can affect the ease of use and reliability of eHealth systems, reducing patients' willingness to transfer their IBD management from the clinic to a technology-based tool and reducing clinicians' willingness to use the technology in the outpatient setting ${ }^{49}$. The minimal evidence available to demonstrate the effectiveness of eHealth interventions in IBD creates uncertainty and hence a reluctance of clinicians and policy makers to implement such technologies ${ }^{24}$. Engagement with end users in the development of the eHealth tool should improve traction 
and sustainability and reduce this uncertainty ${ }^{50}$. Understanding end users' resistance to change will ensure a more successful intervention that is effective, safe, technically robust, accessible and usable.

This study includes limitations that are inherent to qualitative research. The complex influences between the researchers, research questions and the participants may constitute a potential bias during the focus group interview process. Although relatively small, the sample size was adequate for the purpose of this study to inform the development of a decision support tool, especially as a purposeful sampling approach was used in an effort to maximise information gains from key stakeholders and reach thematic saturation. Though the study was conducted at a single center and thus may limit the external validity of the results, a recent quality assurance audit undertaken by CCA indicates that the care provided by our centre is largely commensurate with the care provided by other tertiary IBD centres. We therefore feel that the themes generated by this focus group are generalisable to the majority of tertiary IBD cohorts.

Overall key stakeholders supported the proposed eHealth decision support tool to facilitate IBD self-management. The eHealth tool should provide decision support to patients with stable, mild-to-moderate UC, be based on internationally recognised IBD guidelines and include both PROs and objective markers of inflammatory disease as key outcome measures. A multistage iterative approach should be adopted in the design and implementation process of eHealth interventions. Future studies should include investigations of patient perspectives and controlled testing of eHealth tools. 


\section{REFERENCES}

1. Sawyer SL, Hartley T, Dyment DA, et al. Utility of whole-exome sequencing for those near the end of the diagnostic odyssey: time to address gaps in care. Clin Genet 2016;89:275-84.

2. Jewell DP, Satsangi J, Lobo A, et al. Infliximab use in Crohn's disease: impact on health care resources in the UK. European journal of gastroenterology \& hepatology 2005;17:1047-52.

3. Kassam Z, Belga S, Roifman I, et al. Inflammatory bowel disease cause-specific mortality: a primer for clinicians. Inflammatory bowel diseases 2014;20:2483-92.

4. Huang VW, Reich KM, Fedorak RN. Distance management of inflammatory bowel disease: systematic review and meta-analysis. World journal of gastroenterology : WJG 2014;20:829-42.

5. Walker JR, Ediger JP, Graff LA, et al. The Manitoba IBD cohort study: a population-based study of the prevalence of lifetime and 12-month anxiety and mood disorders. Am J Gastroenterol 2008;103:1989-97.

6. Group TIS. Standards for the Healthcare of People who have Inflammatory Bowel Disease (IBD). 2013.

7. Jess T, Frisch M, Simonsen J. Trends in overall and cause-specific mortality among patients with inflammatory bowel disease from 1982 to 2010. Clinical gastroenterology and hepatology : the official clinical practice journal of the American Gastroenterological Association 2013;11:43-8.

8. Molodecky NA, Soon IS, Rabi DM, et al. Increasing incidence and prevalence of the inflammatory bowel diseases with time, based on systematic review. Gastroenterology 2012;142:46-54 e42; quiz e30. 9. Mikocka-Walus AA, Andrews JM, Bernstein CN, et al. Integrated models of care in managing inflammatory bowel disease: a discussion. Inflamm Bowel Dis;18:1582-7.

10. Mikocka-Walus AA, Turnbull D, Holtmann G, Andrews JM. An integrated model of care for inflammatory bowel disease sufferers in Australia: development and the effects of its implementation. Inflamm Bowel Dis;18:1573-81. 
11. Grone O, Garcia-Barbero M, Services WHOEOfIHC. Integrated care: a position paper of the WHO European Office for Integrated Health Care Services. International journal of integrated care 2001;1:e21.

12. van Langenberg DR, Simon SB, Holtmann GJ, Andrews JM. The burden of inpatient costs in inflammatory bowel disease and opportunities to optimize care: a single metropolitan Australian center experience. Journal of Crohn's \& colitis 2010;4:413-21.

13. Jackson BD, Gray K, Knowles SR, De Cruz P. EHealth Technologies in Inflammatory Bowel Disease: A Systematic Review. Journal of Crohn's \& colitis 2016;10:1103-21.

14. Lorig KR, Holman H. Self-management education: history, definition, outcomes, and mechanisms. Annals of behavioral medicine : a publication of the Society of Behavioral Medicine 2003;26:1-7.

15. Huang VW, Reich KM, Fedorak RN. Distance management of inflammatory bowel disease: systematic review and meta-analysis. World $\mathrm{J}$ Gastroenterol;20:829-42.

16. Barlow C, Cooke D, Mulligan K, Beck E, Newman S. A critical review of self-management and educational interventions in inflammatory bowel disease. Gastroenterology nursing : the official journal of the Society of Gastroenterology Nurses and Associates 2010;33:11-8.

17. Shortliffe EH. Computer programs to support clinical decision making. Jama 1987;258:61-6.

18. Cross RK, Jambaulikar G, Langenberg $P$, et al. TELEmedicine for Patients with Inflammatory Bowel Disease (TELE-IBD): Design and implementation of randomized clinical trial. Contemporary clinical trials 2015;42:132-44.

19. Heida A, Dijkstra A, Groen H, Muller Kobold A, Verkade H, van Rheenen $\mathrm{P}$. Comparing the efficacy of a web-assisted calprotectin-based treatment algorithm (IBD-live) with usual practices in teenagers with inflammatory bowel disease: study protocol for a randomized controlled trial. Trials 2015;16:271.

20. Carlsen K, Houen G, Jakobsen C, et al. Individualized Infliximab Treatment Guided by Patient-managed eHealth in Children and Adolescents with Inflammatory Bowel Disease. Inflammatory bowel diseases 2017. 
21. Pedersen N, Thielsen $P$, Martinsen $L$, et al. eHealth: individualization of mesalazine treatment through a self-managed web-based solution in mild-tomoderate ulcerative colitis. Inflammatory bowel diseases 2014;20:2276-85.

22. de Jong $M$, van der Meulen-de Jong $A$, Romberg-Camps $M$, et al.

Development and Feasibility Study of a Telemedicine Tool for All Patients with IBD: MylBDcoach. Inflammatory bowel diseases 2017;23:485-93.

23. Agboola S, Hale TM, Masters C, Kvedar J, Jethwani K. "Real-world" practical evaluation strategies: a review of telehealth evaluation. JMIR research protocols 2014;3:e75.

24. Pagliari C. Design and evaluation in eHealth: challenges and implications for an interdisciplinary field. Journal of medical Internet research 2007;9:e15.

25. Coulter A, Stilwell D, Kryworuchko J, Mullen PD, Ng CJ, van der Weijden T. A systematic development process for patient decision aids. BMC medical informatics and decision making 2013;13 Suppl 2:S2.

26. Palinkas LA, Horwitz SM, Green CA, Wisdom JP, Duan N, Hoagwood K. Purposeful Sampling for Qualitative Data Collection and Analysis in Mixed Method Implementation Research. Administration and policy in mental health 2015;42:533-44.

27. Braun V, Clarke V. Using thematic analysis in psychology. Qualitative Research in Psychology 2006;3:77-101.

28. Sim J, Wright CC. The kappa statistic in reliability studies: use, interpretation, and sample size requirements. Physical therapy 2005;85:25768.

29. Dolan PL. Participatory medicine: A high-tech alliance with patients. 2010.

30. Kelders DSM. Understanding adherence to web-based interventions. Enschede2012.

31. Altuwaijri MM, Sughayr AM, Hassan MA, Alazwari FM. The effect of integrating short messaging services ' reminders with electronic medical records on non-attendance rates. Saudi Med J 2012;33:193-6.

32. Cross RK, Cheevers N, Rustgi A, Langenberg P, Finkelstein J. Randomized, controlled trial of home telemanagement in patients with ulcerative colitis (UC HAT). Inflammatory bowel diseases 2012;18:1018-25. 
33. Wheaton M, O'Neill S, Brady RR. Colorectal smartphone apps: opportunities and risks. JMIR Mhealth Uhealth 2012;14:e530-4.

34. Con D, De Cruz P. Mobile Phone Apps for Inflammatory Bowel

Disease Self-Management: A Systematic Assessment of Content and Tools. JMIR Mhealth Uhealth 2016;4:e13.

35. Kim ES, Park KS, Cho KB, et al. Development of a Web-based, selfreporting symptom diary for Crohn's Disease, and its correlation with the Crohn's Disease Activity Index: Web-based, self-reporting symptom diary for Crohn's Disease. J Crohns Colitis 2014.

36. Marin-Jimenez I, Nos P, Domenech E, et al. Diagnostic Performance of the Simple Clinical Colitis Activity Index Self-Administered Online at Home by Patients With Ulcerative Colitis: CRONICA-UC Study. The American journal of gastroenterology 2016;111:261-8.

37. Van Deen WK, van der Meulen-de Jong AE, Parekh NK, et al. Development and Validation of an Inflammatory Bowel Diseases Monitoring Index for Use With Mobile Health Technologies. Clinical gastroenterology and hepatology : the official clinical practice journal of the American Gastroenterological Association 2016;14:1742-50 e7.

38. Heida A, Knol M, Kobold AM, Bootsman J, Dijkstra G, van Rheenen PF. Agreement Between Home-based Measurement of Stool Calprotectin and ELISA Results for Monitoring Inflammatory Bowel Disease Activity. Clinical gastroenterology and hepatology : the official clinical practice journal of the American Gastroenterological Association 2017.

39. Vinding KK, Elsberg $\mathrm{H}$, Thorkilgaard $\mathrm{T}$, et al. Fecal Calprotectin Measured By Patients at Home Using Smartphones--A New Clinical Tool in Monitoring Patients with Inflammatory Bowel Disease. Inflammatory bowel diseases 2016;22:336-44.

40. Birnbaum F, Lewis D, Rosen RK, Ranney ML. Patient engagement and the design of digital health. Academic emergency medicine : official journal of the Society for Academic Emergency Medicine 2015;22:754-6.

41. Makai $P$, Melis RJ, Olde-Rikkert MG. Technical difficulties and evaluating e-health interventions. JAMA Intern Med 2014;174:304-5. 
42. Bennett GG, Glasgow RE. The delivery of public health interventions via the Internet: actualizing their potential. Annual review of public health 2009;30:273-92.

43. Ritterband LM, Thorndike FP, Cox DJ, Kovatchev BP, GonderFrederick LA. A behavior change model for internet interventions. Annals of behavioral medicine : a publication of the Society of Behavioral Medicine 2009;38:18-27.

44. Schnall R, Higgins T, Brown W, Carballo-Dieguez A, Bakken S. Trust, Perceived Risk, Perceived Ease of Use and Perceived Usefulness as Factors Related to mHealth Technology Use. Studies in health technology and informatics 2015;216:467-71.

45. Webb TL, Joseph J, Yardley L, Michie S. Using the internet to promote health behavior change: a systematic review and meta-analysis of the impact of theoretical basis, use of behavior change techniques, and mode of delivery on efficacy. Journal of medical Internet research 2010;12:e4.

46. Holden RJ, Karsh BT. The technology acceptance model: its past and its future in health care. Journal of biomedical informatics 2010;43:159-72.

47. Anderson R. New MRC guidance on evaluating complex interventions. Bmj 2008;337:a1937.

48. van Mierlo T, Fournier R. Don't Forget the Doctor: Gastroenterologists' Preferences on the Development of mHealth Tools for Inflammatory Bowel Disease. 2015;3:e5.

49. Dumay AC, Freriks G. Quality management issues for medical ICT. Studies in health technology and informatics 2004;103:93-100.

50. McCurdie T, Taneva S, Casselman M, et al. mHealth consumer apps: the case for user-centered design. Biomedical instrumentation \& technology 2012;Suppl:49-56. 


\section{TABLES}

Table 1. Demographics of the participants of the focus group.

\begin{tabular}{ll}
\hline Demographic & $n$ \\
\hline Gender & 16 \\
Male & 15 \\
Female & \\
Position & 10 \\
Gastroenterologist (physician) & 1 \\
Non-gastroenterology physician & 6 \\
Nurse consultant & 2 \\
Hospital executive & 1 \\
Pharmacist & 3 \\
Medical/PhD student & 1 \\
IBD Surgeon & 1 \\
Crohn's Colitis Australia representative & 1 \\
Outpatient coordinator & 1 \\
Patient Flow Director & 1 \\
Head of IT Services & 2 \\
Medical researcher & 1 \\
General Practitioner Liaison &
\end{tabular}

This article is protected by copyright. All rights reserved. 
Table 2. Key themes with exemplar quotes.

\begin{tabular}{ll}
\hline Suggestion & Exemplar quote \\
\hline $\begin{array}{ll}\text { 1. Framework } \\
\text { Alternative model of chronic disease }\end{array}$ & "Swollen wait lists—six months; cost per patient is \\
management is needed & enormous; frustration; long wait times et cetera; they're \\
& really interested in eHealth resources." \\
eHealth as a participatory health & "I think that's (eHealth) actually very successful, it certainly \\
care model to augment existing & incorporates this participatory care, defined by the 'patient \\
management & knows best'." \\
& "People want this thing already."
\end{tabular}

\section{Target population}

Stable disease patients

"People who have had the diagnosis for at least two years, stable for a year."

"Patients escalation and de-escalation steroids would be a particularly challenging area."

Mild-to-moderate disease severity "Only stable patients with mild-to-moderate UC should be included."

\section{Functionalities}

Web-based platform

"We should minimize the content of email and move people into a web-based interface as quickly as we can."

Disease monitoring using clinical algorithms from ECCO guidelines

Psychological management "The treatment algorithms should be derived from the ECCO guidelines."

"I think it's actually identifying that they're not managingthat they're not coping."

Decision support "Patient's ability to self-manage their IBD seems feasible."

Patient reported outcomes "I think that PROs is a key, though."

\section{Design and implementation}

Patient engagement in the design process

Usability and sustainability

"Participatory design and evaluation is increasingly highly regarded. To get representatives of the community, right through the whole process is really essential."

"I think especially, you've got to make your IT really slick...if it's clunky and difficult, people are going to lose interest quickly."

"Patients' feedback is that the more visually engaging, the less effort it takes from the patient's perspective." 


\section{University Library}

\section{- M M N E R VA A gateway to Melbourne's research publications}

Minerva Access is the Institutional Repository of The University of Melbourne

Author/s:

Jackson, BD;Con, D;De Cruz, P

Title:

Design considerations for an eHealth decision support tool in inflammatory bowel disease self-management

Date:

2018-06-01

Citation:

Jackson, B. D., Con, D. \& De Cruz, P. (2018). Design considerations for an eHealth decision support tool in inflammatory bowel disease self-management. INTERNAL MEDICINE JOURNAL, 48 (6), pp.674-680. https://doi.org/10.1111/imj.13677.

Persistent Link:

http://hdl.handle.net/11343/283435 